\title{
EDITORIAL
}

\section{Two years of COVID-19 pandemic: where are we now?}

\author{
Jinjong Myoung ${ }^{*}$ \\ Korea Zoonosis Research Institute, Department of Bioactive Material \\ Science and Genetic Engineering Research Institute, Jeonbuk National \\ University, Jeonju 54531, Republic of Korea
}

Unprecedented. This is the closest and most appropriate word to describe the COVID-19 pandemic, which the world has been experiencing with pain and fear. The first case of pneumonia-like symptoms of unknown etiology appeared presumably in November 2019, with the subsequent official report to the WHO by the Chinese authorities on December 31, 2019. China's first confirmed death from the virus occurred on January 11, 2020, when a 61-year-old male resident of Hubei, the capital of Wuhan Province, died. Within a month, the COVID-19 death toll surpassed 1,000 (February 10, 2020). Accordingly, just 30 days after the initial report, the coronavirus outbreak was called a "public health emergency of international concern" by the WHO, the organization's highest alert level. Unfortunately, the WHO soon declared the COVID-19 outbreak a pandemic (March 11, 2020). Within a year of viral emergence, and by December 2020, more than 80 million confirmed cases had been reported worldwide. Infections increased exponentially over the following year. As of February 11, 2022, over 400 million cases have been reported, with nearly 6 million deaths, an unprecedented rate of spread across borders.

\section{SARS-CoV-2 Virology and Variant Emergence}

The causative agent was identified on January 7, 2020, only seven days after the initial report of "pneumonia-like diseases" in Wuhan. It was first named "novel coronavirus" (nCoV) and later officially classified as severe acute respiratory syndrome coronavirus 2 (SARS-CoV-2) by the International Committee on Taxonomy of Viruses on February 11, 2020. Genome analysis revealed that the virus had $79 \%$ homology to the severe acute respiratory syndrome coronavirus (SARS-CoV) and approximately $50 \%$ homology to the Middle East respiratory syndrome coronavirus (MERS-CoV). Due to the high structural similarity between SARS- CoV and SARS-CoV-2 spike proteins, angiotensin-converting enzyme 2 (ACE2), which is also the receptor of SARS- CoV, was soon identified as the putative receptor of SARS-CoV-2. The discovery of the cel-

*For correspondence. E-mail: jinjong.myoung@jbnu.ac.kr; Tel.: +82-639004055; Fax: +82-63-9004012

Copyright (c) 2022, The Microbiological Society of Korea lular receptor triggered a flurry of research efforts to develop virus-specific antivirals, such as soluble ACE2 fused to the Fc region of immunoglobulin and monoclonal antibodies that disrupt interactions between the viral spike and cellular ACE2 proteins, thus inhibiting viral entry into the host cell.

On the other hand, a number of studies have focused on understanding how SARS-CoV-2 interacts with the host innate immune system, which is the first line of defense against invading pathogens. Pathogen-associated molecular patterns (PAMPs), such as viral genomes and protein components of virus particles, are recognized by several pattern recognition receptors (PRRs) in the cytoplasm or on the membrane: retinoic acid-induced gene I (RIG-I)-like receptors (RLRs), tolllike receptors (TLRs), and NOD-like receptors (NLRs). Binding of PAMPs to PRRs triggers a cascade of activation events of cellular signaling proteins, culminating in interferon (IFN) and interferon-stimulated gene (ISG) expression. IFNs and ISGs inhibit viral gene expression, genome amplification, viral assembly, and/or viral egress. Thus, SARS-CoV-2 must evade the host innate immunity for the successful establishment of viral infection and replication inside cells. SARS$\mathrm{CoV}-2$ genome encodes many molecules involved in immune evasion mechanisms (Kim and Song, 2022; Oh and Shin, 2022). Accessory proteins of coronaviruses play pivotal roles in immune evasion. Interestingly, in addition to accessory proteins, multiple structural and non-structural proteins participate in host innate immunity evasion as well. However, as most of these studies employed only transient viral gene overexpression, defining detailed inhibitory mechanisms and their relative importance in immune evasion awaits further scrutiny in the context of viral infections.

Cell and animal infection models are required to investigate the pathophysiology of SARS-CoV-2 infection. After the identification of ACE2 as a cellular receptor for SARS-CoV2 , in silico studies revealed that the mouse homolog sequences only weakly bind to the viral spike protein. Thus, it was predicted that normal mice would not be susceptible to SARSCoV-2 infection. When human ACE2 (hACE2)-transgenic mice (hACE2-Tg) were infected, they displayed high levels of viral infection, replication, and body weight loss. However, interestingly, infected mice died of brain damage rather than lung pathologies, which does not mimic the human pathology upon SARS-CoV-2 infection. Although hACE2-Tg may not serve as a pathological model of viral infection, it is an excellent model for vaccine efficacy testing, with subsequent challenge with pathogenic viruses. Other animal models, such as hamsters, ferrets, and monkeys have also been used (Kim et al., 2022a). With the emergence of SARS-CoV-2 variants, 
an interesting twist in infection models was evident. Most variants have extensively mutated the spike protein sequences to evade the antibody responses of the host. Some mutations enable the virus to bind to mouse ACE2, thus rendering normal mice susceptible to viral infection. A prominent example of this is the Beta variant. SARS-CoV-2 continues to change by mutations, expanding its territory in the animal kingdom.

\section{Vaccines and Therapeutics against SARS-CoV-2}

The unprecedented crisis prompted unprecedented global collaborations to create effective SARS-CoV-2 countermeasures, including vaccines and treatments. The development of therapeutics has made some early strides thanks to socalled "drug repositioning approaches." On March 30, 2020, the FDA issued an emergency use authorization (EUA) for donating "hydroxychloroquine sulfate and chloroquine phosphate products" to the Strategic National Stockpile and hospitals to treat COVID-19 patients. However, in light of complaints of heart rhythm difficulties in some patients, the EUA was revoked on June 15, 2020. A large clinical trial of postexposure therapy with hydroxychloroquine concluded that hydroxychloroquine does not prevent SARS-CoV-2 infection or symptomatic COVID-19. Later on June 18, 2020, the WHO announced that it would suspend evaluating hydroxychloroquine as a COVID-19 medication since the "Solidarity Trial" found no indication that the medicine reduced mortality. It was somewhat disappointing, given former US President Donald Trump's high level of excitement in the drug. Remdesivir (Gilead Sciences), a hepatitis and Ebola medicine, was the next therapeutic candidate to receive media attention. On May 1, 2020, remdesivir was approved by the US Food and Drug Administration for use in treating COVID-19, as the drug was expected to reduce clinical symptoms. However, subsequent studies have questioned its clinical benefits. Remdesivir seemed to have minimal effect on hospitalized COVID-19 patients according to a global, multicenter study published in JAMA on August 24, 2020. On October 22, 2020, the tide changed again with the FDA approving remdesivir as the first COVID-19 medicine after three randomized trials revealed that it shortens hospital stays and reduces the likelihood of patients requiring oxygen, although none of the trials demonstrated a reduced mortality risk. Molnupiravir, a prodrug of the synthetic nucleoside originally developed at Emory University for treating influenza, was the third excitement to arrive and depart. In December 2021, the FDA approved EUA for using molnupiravir in select populations where other treatments were not practicable, based on its significant but not dramatic effectiveness in placebo-controlled double-blind randomized clinical trials. When paxlovid (nirmatrelvir/ritonavir), an oral antiviral, was introduced in December 2021, there was a lot of enthusiasm. Paxlovid administration within five days of the onset of symptoms for treating mild-to-moderate COVID-19 has $88 \%$ efficacy against hospitalization or mortality in adults. A drawback of paxlovid is the possibility of major adverse medication responses. This medicine is not recommended for people with hypersensitivity to the two primary ingredients or substantially impaired kidney or liver function. Therefore, the development of a safer and more effective drug is desirable. Scores of therapeutic candidates are currently under development. What is lacking in the list are natural products or their derivatives that are accessible and affordable, particularly for the coming era of endemic when the current pandemic is settled (Park et al., 2022b).

While virus-specific antivirals are still in development, major triumphs over SARS-CoV-2 have come from the field of vaccines. Safety and effectiveness are the two crucial characteristics that an ideal vaccine must have. It is also noteworthy that successful development of highly effective COVID-19 vaccines was based on prior research experience and knowhow acquired during the development of other viral vaccines. In terms of vaccine safety, antibody-dependent enhancement (ADE), which has impeded the development of effective vaccines against dengue virus (Park et al., 2022a) and SARS-CoV1 , was a major potential downside that a successful COVID19 vaccine must avoid. Fortunately, while some antibodies generated by vaccinations/infections may induce ADE in vitro, they did not cause any disease in vivo, which came as a great relief. In terms of vaccine efficacy, several studies suggest that stabilizing the pre-fusion conformation of the spike protein by introducing two proline substitutions considerably boosted antibody responses against MERS-CoV and SARS$\mathrm{CoV}$, the two SARS-CoV-2 relatives (Choi and Kim, 2022). Thus, most vaccine immunogens for SARS-CoV-2 have accordingly adopted the strategy of pre-fusion stabilization.

The first COVID-19 vaccine trial began on April 23, 2020, in the UK, by an Oxford University team in Europe (chimpanzee adenovirus-based expression of spike proteins). In July 2020, Moderna, the first company to begin a human study of the COVID-19 vaccine (in the mRNA platform), released preliminary findings indicating that it elicited an immune response against the virus with no major adverse effects. In November 2020, Pfizer announced that its vaccine candidate in the mRNA platform developed with BioNTech was more than $90 \%$ effective in preventing COVID-19 in participants without evidence of past SARS-CoV-2 infection in the first interim effectiveness investigation. On December 8, 2020, a 90 -year-old woman from the UK became the world's first individual to receive the COVID-19 vaccine. After a flurry of breath-taking competitions and collaborations aimed at creating COVID-19 vaccines, the WHO issued an emergency use validation for the Pfizer-BioNTech COVID-19 vaccine on December 31,2020, exactly one year after China's initial report of COVID-19. Despite their excellent effectiveness with a quick development cycle and minor side effects (Park et al., 2022c), these three vaccines have a number of disadvantages, including the levels of neutralizing antibodies decreasing rapidly over time, generating mostly humoral immune responses that lose effectiveness against SARS-CoV-2 variants, and eliciting little to no IgA responses critical for preventing viral infections in mucosal tissues. Therefore, exploring vaccines based on other platforms, such as bacterial and viral vector vaccines (Lee and Kim, 2022), as well as virus-like particle and nanoparticle vaccines (Kim et al., 2022b), might help address some of the flaws in current COVID-19 vaccines. Another interesting and potentially powerful strategy to maximize the efficacy of currently available vaccines is heterologous prime-boosting vaccination, which mixes and 
matches different vaccines. Its efficacy and safety have also been investigated (Mattoo and Myoung, 2021). To design and develop a desirable vaccine, it is also of great importance to remember that vaccine-induced antibody responses might have undesirable disease-exacerbating roles as well. Understanding both the protective and pathogenic roles of human immune responses should be taken into account for the preparation of a better vaccine (Park and Cho, 2022). In addition, the ever-changing nature of viruses, emergence of vaccine-escaping SARS-CoV-2 variants, and the downside of heavy dependence of vaccines on antibody responses alone prompted the analysis of harder-to-escape host immune responses: T cell immunity (Mattoo and Myoung, 2022), which might help pave the way to develop efficient and 'universal' vaccines against SARS-CoV-2.

\section{Conclusion}

With the advent of the Omicron variant, the coronavirus pandemic saga has taken its critical twist on the verge of ending of the pandemic or begining of a new one. Although its clinical symptoms seem milder than those of other variants (closer to those of seasonal flu), it has risen to the top of the authorities' priority list for monitoring because of its high transmissibility and ability to escape previous infection- or immunization-induced immune responses. What if Omicron infections cause a tremendous patient influx, leading to the failure of the medical system? What if the recombination of omicrons with other variants, such as delta, generate a highly pathogenic variant?

To avoid a potential catastrophe caused by a new variant, we must first understand where we are now and how we will proceed to properly prepare ourselves for the impending headwind.

\section{References}

Choi, J. and Kim, J.O. 2022. Middle East Respiratory Syndrome coronavirus vaccine development: updating clinical studies using platform technologies. J. Microbiol. 60, 238-246.

Kim, Y.I., Casel, M.A.B., and Choi, Y.K. 2022a. Transmissibility and pathogenicity of SARS-CoV-2 variants in animal models. J. Microbiol. 60, 255-267.

Kim, C., Kim, J.D., and Seo, S.U. 2022b. Nanoparticle and virus-like particle vaccine approaches against SARS-CoV-2. J. Microbiol. 60, 335-346.

Kim, N.E. and Song, Y.J. 2022. Coordinated regulation of interferon and inflammasome signaling pathways by SARS-CoV-2 proteins. J. Microbiol. 60, 300-307.

Lee, M.H. and Kim, B.J. 2022. COVID-19 vaccine development based on recombinant viral and bacterial vector systems: combinatorial effect of adaptive and trained immunity. J. Microbiol. 60, 321334.

Mattoo, S.S. and Myoung, J. 2021. A promising vaccination strategy against COVID-19 on the horizon: heterologous immunization. J. Microbiol. Biotechnol. 31, 1601-1614.

Mattoo, S.S. and Myoung, J. 2022. T cell responses to SARS-CoV2 in humans and animals. J. Microbiol. 60, 276-289.

Oh, S.J. and Shin, O.S. 2022. SARS-CoV-2-mediated evasion strategies for antiviral interferon pathways. J. Microbiol. 60, 290-299.

Park, U. and Cho, N.H. 2022. Protective and pathogenic role of humoral responses in COVID-19. J. Microbiol. 60, 268-275.

Park, J., Kim, J., and Jang, Y.S. 2022a. Current status and perspectives on vaccine development against dengue virus infection. $J$. Microbiol. 60, 247-254.

Park, J., Park, R., Jang, M., Park, Y.I., and Park, Y. 2022b. Coronavirus enzyme inhibitors-experimentally proven natural compounds from plants. J. Microbiol. 60, 347-354.

Park, H., Park, M.S., Seok, J.H., You, J., Kim, J., Kim, J., and Park, M.S. 2022 c. Insights into the immune responses of SARS-CoV-2 in relation to COVID-19 vaccines. J. Microbiol. 60, 308-320. 